\title{
Star Formation Under the Outflow: The Discovery of a Non-thermal Jet from OMC-2 FIR 3 and Its Relationship to the Deeply Embedded FIR 4 Protostar
}

\author{
Mayra Osorio $^{1}$, Ana K. Díaz-Rodríguez ${ }^{1}$, Guillem Anglada ${ }^{1}$, S. Thomas Megeath ${ }^{2}$, Luis F. Rodríguez ${ }^{3}$, John J. Tobin ${ }^{4,12}$, \\ Amelia M. Stutz ${ }^{5}$, Elise Furlan ${ }^{6}$, William J. Fischer ${ }^{7}$, P. Manoj ${ }^{8}$, José F. Gómez ${ }^{1}$, Beatriz González-García ${ }^{9,13}$, Thomas Stanke ${ }^{10}$, \\ Dan M. Watson ${ }^{11}$, Laurent Loinard ${ }^{3,14}$, Roland Vavrek $^{9}$, and Carlos Carrasco-González ${ }^{3}$ \\ ${ }^{1}$ Instituto de Astrofísica de Andalucía (CSIC) Glorieta de la Astronomía s/n E-18008 Granada, Spain; osorio@iaa.es \\ ${ }^{2}$ Ritter Astrophysical Research Center, Department of Physics and Astronomy University of Toledo 2801 West Bancroft Street Toledo, OH 43606, USA \\ 3 Instituto de Radioastronomía y Astrofísica, UNAM Apartado Postal 3-72 (Xangari), 58089 Morelia, Michoacán, Mexico \\ ${ }^{4}$ Homer L. Dodge Department of Physics and Astronomy University of Oklahoma, Norman, OK 73019, USA \\ ${ }^{5}$ Department of Astronomy, University of Concepción Concepción, Chile \\ ${ }^{6}$ IPAC, Mail Code 314-6, Caltech 1200 E. California Boulevard, Pasadena, CA 91125, USA \\ ${ }^{7}$ Space Telescope Science Institute 3700 San Martin Drive, Baltimore, MD 21218, USA \\ ${ }^{8}$ Tata Institute of Fundamental Research Homi Bhabha Road, Mumbai 400 005, India \\ ${ }^{9}$ European Space Astronomy Center, ESA P.O. Box 78, E-28691 Villanueva de la Cañada, Madrid, Spain \\ ${ }^{10}$ European Southern Observatory Garching bei München, Germany \\ ${ }^{11}$ Department of Physics and Astronomy, University of Rochester Rochester, NY 14627, USA \\ Received 2017 February 12; revised 2017 March 22; accepted 2017 March 22; published 2017 May 3
}

\begin{abstract}
We carried out multiwavelength $(0.7-5 \mathrm{~cm})$, multi-epoch (1994-2015) Very Large Array (VLA) observations toward the region enclosing the bright far-IR sources FIR 3 (HOPS 370) and FIR 4 (HOPS 108) in OMC-2. We report the detection of 10 radio sources, 7 of them identified as young stellar objects. We image a well-collimated radio jet with a thermal free-free core (VLA 11) associated with the Class I intermediate-mass protostar HOPS 370. The jet features several knots (VLA 12N, 12C, 12S) of non-thermal radio emission (likely synchrotron from shock-accelerated relativistic electrons) at distances of $\sim 7500-12,500$ au from the protostar, in a region where other shock tracers have been previously identified. These knots are moving away from the HOPS 370 protostar at $\sim 100 \mathrm{~km} \mathrm{~s}^{-1}$. The Class 0 protostar HOPS 108, which itself is detected as an independent, kinematically decoupled radio source, falls in the path of these non-thermal radio knots. These results favor the previously proposed scenario in which the formation of HOPS 108 is triggered by the impact of the HOPS 370 outflow with a dense clump. However, HOPS 108 has a large proper motion velocity of $\sim 30 \mathrm{~km} \mathrm{~s}^{-1}$, similar to that of other runaway stars in Orion, whose origin would be puzzling within this scenario. Alternatively, an apparent proper motion could result because of changes in the position of the centroid of the source due to blending with nearby extended emission, variations in the source shape, and/or opacity effects.
\end{abstract}

Key words: ISM: jets and outflows - proper motions - radio continuum: stars - stars: formation - stars: individual (OMC-2 FIR 3, HOPS 370, OMC-2 FIR 4, HOPS 108) - stars: protostars

\section{Introduction}

OMC-2 is an active star-forming region (e.g., Peterson \& Megeath 2008) in the Orion A molecular cloud, located at a distance of $414 \pm 7 \mathrm{pc}$ (Menten et al. 2007; Kim et al. 2008; Kounkel et al. 2017). Mezger et al. (1990) identified six bright millimeter/IR sources (FIR 1-6) within a region of about $6^{\prime}$ in size that have been associated with young stellar objects (YSOs) through subsequent studies (Adams et al. 2012; Furlan et al. 2014, 2016 and references therein). The region has been imaged at millimeter and submillimeter wavelengths by Chini et al. (1997) and Lis et al. (1998), and in the near- and mid-IR by Tsujimoto et al. (2003), Nielbock et al. (2003), and Megeath et al. (2012). At millimeter and submillimeter wavelengths the brightest source is FIR 4, which has been associated with the HOPS 108 Class 0 protostar (Adams et al. 2012; Furlan et al. 2016). ${ }^{15}$ This source is connected through a filamentary cloud structure to the

\footnotetext{
12 Leiden Observatory, Leiden University, P.O. Box 9513, 2300-RA Leiden, The Netherlands.

13 ISDEFE, Beatriz de Bobadilla 3, E-28040 Madrid, Spain.

14 Instituto de Astronomía, UNAM, Apartado Postal 70-264, CDMX C.P. 04510, Mexico.

15 In low-resolution observations FIR 4 probably includes emission from other nearby objects.
}

bright source FIR 3, also known as HOPS 370, an intermediatemass Class I YSO with an $L_{\mathrm{bol}} \sim 360 L_{\odot}$ (Adams et al. 2012, Furlan et al. 2016) located about $30^{\prime \prime}$ to the NE (see Figure 1 for the positions and nomenclature of the sources). The region was observed with the Very Large Array (VLA) at $3.6 \mathrm{~cm}$ in the D configuration (angular resolution $\sim 8^{\prime \prime}$ ) by Reipurth et al. (1999). These authors found a weak elongated source (VLA 12) toward FIR 4 (HOPS 108) that was interpreted as a radio jet originating from this protostar. A stronger but unresolved source (VLA 11) was detected toward FIR 3 (HOPS 370).

The nature of the source FIR 4 is still uncertain. Shimajiri et al. (2008), through millimeter and submillimeter observations proposed that FIR 4 was composed of 11 dusty cores. These authors detected molecular line emission from shock tracers $(\mathrm{SiO}$ and $\left.\mathrm{CH}_{3} \mathrm{OH}\right)$ in the proximity of FIR 4 and proposed that the interaction of a powerful molecular outflow driven by FIR 3 (e.g., Williams et al. 2003, Takahashi et al. 2008) with a dense clump could be triggering the formation of the next generation of stars at the position of FIR 4. On the other hand, López-Sepulcre et al. (2013), based on $2 \mathrm{~mm}$ observations, proposed that FIR 4 was composed of at least three cores, and suggested that its associated $3.6 \mathrm{~cm}$ source VLA 12 traces an H II region photoionized by an early-type (B3-B4) star with a luminosity of $700-1000 L_{\odot}$ 

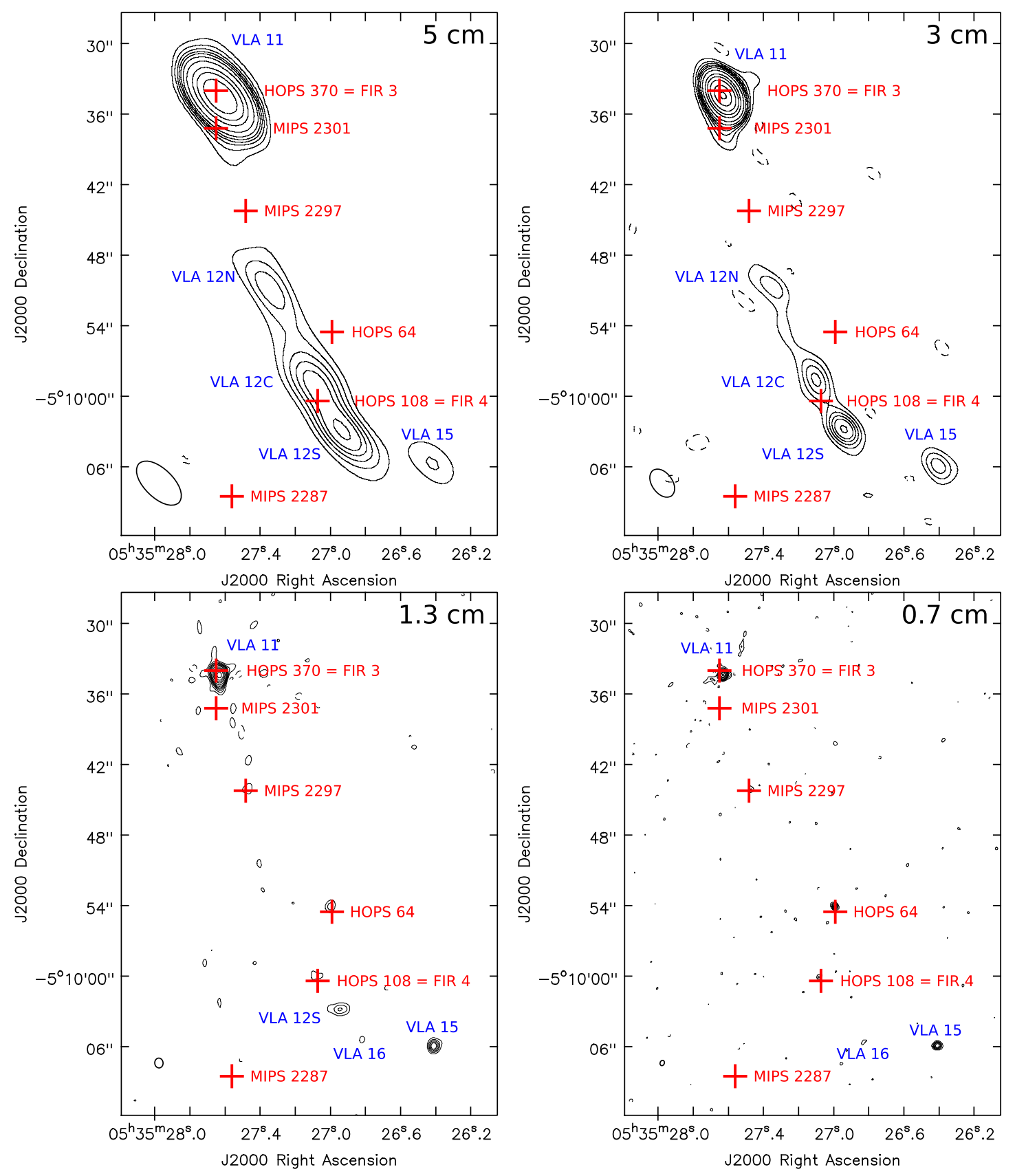

Figure 1. VLA C-configuration maps (epoch 2014) of the region around the HOPS 108 protostar. Contour levels are $-3,3,6,9,12,15,20$, 30, 50, 70, 100, 150, and 200 times the rms noise of each map. The robust weighting parameter (Briggs 1995) was set to 0 . The synthesized beam is shown in the bottom left corner of each map. The $10.4 \mu \mathrm{m}$ positions (Nielbock et al. 2003) are indicated by red plus signs whose sizes correspond to the positional uncertainty. For HOPS 108, which is not reported at $10.4 \mu \mathrm{m}$, the $24 \mu \mathrm{m}$ position (Megeath et al. 2012) is given. Top left: $C$ band $(5 \mathrm{~cm}) ; \mathrm{rms}=11 \mu \mathrm{Jy} \mathrm{beam}^{-1} ; \mathrm{HPBW}=4 ! \cdot 75 \times 22^{\prime \prime} 46, \mathrm{P} . \mathrm{A} .=46^{\circ}$. Top right: $X$ band $(3 \mathrm{~cm}) ; \mathrm{rms}=9 \mu \mathrm{Jy}$ beam $^{-1}$; HPBW $=2^{\prime \prime} .68 \times 1^{\prime \prime} .73$, P.A. $=36^{\circ}$. Bottom left: $K$ band $(1.3 \mathrm{~cm}) ; \mathrm{rms}=10 \mu \mathrm{Jy}$ beam ${ }^{-1} ; \mathrm{HPBW}=0{ }^{\prime \prime} 84 \times 00^{\prime \prime} 71$, P.A. $=5^{\circ}$. Bottom right: $Q$ band $(0.7 \mathrm{~cm})$ map; $\mathrm{rms}=15 \mu \mathrm{Jy}$ beam $^{-1}$; HPBW $=0$ !' $46 \times 0$ ". 36, P.A. $=-20^{\circ}$.

embedded within one of these cores. A bolometric luminosity $L_{\text {bol }} \simeq 1000 L_{\odot}$ for FIR 4 was obtained by Crimier et al. (2009), but this estimate, based on low angular resolution IRAS data, is very uncertain because of the difficulties in separating the emission of the protostar from that of the surrounding molecular cloud and other neighboring objects.

In a rigorous analysis of the available photometry of HOPS 108, including Herschel measurements that constrain the peak of the Spectral Energy Distribution (SED) better than the IRAS fluxes, Furlan et al. (2014) give $L_{\mathrm{bol}}=37 L_{\odot}$. This is much lower than the earlier estimate by Crimier et al. (2009) or the value adopted by López-Sepulcre et al. (2013). Due to uncertainties in the amount of external heating and the inclination of the protostar, Furlan et al. (2014) found that they can fit the SED using models with values for the intrinsic total luminosity ${ }^{16}$ ranging from $15 L_{\odot}$ to $540 L_{\odot}$, although models with luminosities $\leqslant 100 L_{\odot}$ are favored.

\footnotetext{
${ }^{16}$ The intrinsic total luminosity of a source and its bolometric luminosity derived from the observed flux densities can differ in the case of an inhomogeneous surrounding medium (e.g., if outflow cavities are present), resulting in an anisotropic radiation field where the observed flux density depends on inclination angle (see Furlan et al. 2016).
} 
Manoj et al. (2013) detected far-IR CO lines indicative of shock-heated gas at high temperatures ( $\gtrsim 2000 \mathrm{~K})$ toward both HOPS 108 and HOPS 370. Recently, González-García et al. (2016) imaged in far-IR [O I] lines and in the submillimeter $\mathrm{CO}(6-5)$ line, a powerful $\left(\dot{M} \simeq 2 \times 10^{-6} M_{\odot} \mathrm{yr}^{-1}\right.$, as estimated by these authors) bipolar jet/outflow originating from HOPS 370. The outflow extends to the NE of HOPS 370 (FIR 3) in the direction where a $4.5 \mu \mathrm{m}$ Spitzer/IRAC image (which traces shock-excited $\mathrm{H}_{2}$ emission) shows an extended jet that terminates in a bow shock (Megeath et al. 2012). To the southwest, the outflow terminates near the location of HOPS 108, with a bright [O I] emission peak that González-García et al. (2016) argue originates in the terminal shock produced by the jet.

In summary, Shimajiri et al. (2008), López-Sepulcre et al. (2013), and González-García et al. (2016) suggest that the protostar HOPS 108 results from the interaction of an outflow driven by HOPS 370 with a dense clump. Within this scenario, López-Sepulcre et al. (2013) assume that a star cluster is being formed, with the radio source VLA 12 tracing an $\mathrm{H}$ II region associated with the most massive star; for this star they adopt a luminosity of $\sim 1000 L_{\odot}$, similar to earlier estimates of Crimier et al. (2009). On the other hand, Furlan et al. (2014), Reipurth et al. (1999), and Adams et al. (2012) interpret VLA 12 as a radio jet driven by a modest luminosity protostar associated with HOPS 108.

In this paper we analyze new and archival VLA observations that shed new light on the origin of the HOPS 108 protostar, the nature of its radio emission, and its relationship with other nearby sources.

\section{Observations}

The observations were carried out with the Karl G. Jansky VLA of the National Radio Astronomy Observatory (NRAO) ${ }^{17}$ in the C-configuration in 2014 (Project 14B-296) at the $C$ $(5 \mathrm{~cm}), X(3 \mathrm{~cm}), K(1.3 \mathrm{~cm})$, and $Q(0.7 \mathrm{~cm})$ bands, and in the A-configuration in 2015 (Project 15A-369) at the $C$ band $(5 \mathrm{~cm})$. In both cases, the phase center was close to the position of HOPS 108. Further details of the observational setup are given in Table 1. The data were edited and calibrated using the Common Astronomy Software Applications (CASA; version 4.2.2) package.

We also analyzed several epochs of VLA archival data at the $X$ band $(3.6 \mathrm{~cm})$ that were obtained with different configurations between 1994 and 2000 (see Table 1 for details). These data were edited and calibrated using the Astronomical Image Processing System (AIPS), and then were concatenated using the CASA package.

All the images were made with the CASA task CLEAN using multi-frequency synthesis (Conway et al. 1990), and by fitting the frequency dependence of the emission with a Taylor series expansion with nterms $=2$ during the deconvolution. There is extended emission of the bright $\mathrm{H}$ II region M43 (NGC 1982) $\sim 5^{\prime}$ to the south of HOPS 108 that makes it difficult to image the archive ( $X$ band) data and the new data at the $C$ band. In order to do a proper cleaning of our target sources we downweighted the extended emission by removing the shortest baselines. For the archive and the $2014 C$ band data we used only baselines $>5 \mathrm{k} \lambda$ and the multiscale deconvolution

\footnotetext{
17 NRAO is a facility of the National Science Foundation operated under cooperative agreement by Associated Universities, Inc.
}

algorithm to make the images. For the 2015 A-configuration data, which are more sensitive, we used only baselines $>25 \mathrm{k} \lambda$.

\section{Results and Discussion}

In our new data of epochs 2014 and 2015 we detect the radio sources VLA 11, VLA 12, and VLA 13, which were previously reported at $3.6 \mathrm{~cm}$ by Reipurth et al. (1999); our observations cover additional bands and are more sensitive, revealing further details. The FWHM of our primary beam, centered near VLA 12 , ranges from $\sim 1^{\prime}$ at the $Q$ band to $\sim 8^{\prime}$ at the $C$ band. Therefore, we detected additional sources, including several of the IR sources in the field. In this paper we will discuss only the sources detected in the proximity (within $\lesssim 30^{\prime \prime}$ ) of HOPS 108 . Positions of these sources are given in Table 2. The remaining detected sources will be discussed in a forthcoming paper (A. K. Díaz-Rodríguez et al. 2017, in preparation).

\subsection{Identification of the Detected Sources}

In Figure 1 we show the maps of VLA 11 and VLA 12 at different wavelengths obtained in our 2014 VLA C-configuration observations. VLA 12 (in the proximity of FIR 4/HOPS 108) is clearly detected as a very elongated source at 5 and $3 \mathrm{~cm}$, showing three knots that we call VLA $12 \mathrm{~N}$, VLA $12 \mathrm{C}$, and VLA $12 \mathrm{~S}$, for the northern, central, and southern observed emission peaks, respectively. At first glance, these results appear to confirm VLA 12 as a radio jet, as was first suggested by Reipurth et al. (1999). These knots coincide with the southern, brightest part of the jet/outflow traced by the far-IR [O I] and submillimeter $\mathrm{CO}(6-5)$ lines, which is assumed to originate in HOPS 370 (González-García et al. 2016; see Figure 2, left). The IR position of the HOPS 108 protostar (Megeath et al. 2012) falls close to (within $\sim 2^{\prime \prime}$ ) the position of knot VLA 12C, apparently favoring HOPS 108 as the driving source of the proposed VLA 12 radio jet.

At shorter wavelengths $(1.3$ and $0.7 \mathrm{~cm}$; bottom panels in Figure 1) the emission of the VLA 12 knots decreases, making them undetectable, indicating that they have a negative spectral index $\alpha$ (where $S_{\nu} \propto \nu^{\alpha}$ ), characteristic of non-thermal emission. This is confirmed by the analysis of the spectra obtained from our full data set (Table 3, Figure 3). Despite the possible time variability both in flux density (that can be more prominent in non-thermal emission) and morphology, as well as the difficulties inherent in the measure of extended emission from data taken with different angular resolutions, the obtained spectral indices in the centimeter range are negative for the three knots of VLA 12 , with values of $-1.1,-1.3$, and -0.6 . The behavior is clear even if only the C-configuration data from 2014 October, which were taken almost simultaneously, are considered. Also, we have checked in these data that the negative spectral indices obtained are not spurious results of differences in the uv coverage and/or angular resolution, that could make data at shorter wavelengths less sensitive to extended emission. To do that, we obtained pairs of images of contiguous bands using only baselines in the same uvrange. Using these pairs of maps we confirmed that the flux density truly decreases at shorter wavelengths, resulting in negative spectral indices for the VLA 12 knots. These negative spectral indices cannot be explained by thermal emission (see Rodríguez et al. 1993) and should be attributed to non-thermal emission, probably arising from relativistic electrons accelerated in strong shocks (Carrasco-González et al. 2010; see 
Table 1

Parameters of the VLA Observations

\begin{tabular}{|c|c|c|c|c|c|c|c|c|c|c|c|c|}
\hline \multirow[b]{3}{*}{ Date } & \multirow{3}{*}{$\begin{array}{l}\text { VLA } \\
\text { Conf. }\end{array}$} & \multirow[b]{3}{*}{ Band } & \multirow{3}{*}{$\begin{array}{c}\text { Central } \\
\text { Frequency } \\
(\mathrm{GHz})\end{array}$} & \multirow{3}{*}{$\begin{array}{l}\text { Bandwidth } \\
\text { (GHz) }\end{array}$} & \multirow[b]{3}{*}{ Project } & \multirow{2}{*}{\multicolumn{2}{|c|}{ Phase Center }} & \multicolumn{3}{|c|}{ Adopted } & \multirow{3}{*}{$\begin{array}{l}\text { Bootstrapped } \\
\text { Flux Density } \\
\text { (Jy) }\end{array}$} & \multirow{3}{*}{$\begin{array}{c}\text { On-Source } \\
\text { Time } \\
\text { (hours) }\end{array}$} \\
\hline & & & & & & & & & Flux Density & Phase & & \\
\hline & & & & & & $\alpha(\mathrm{J} 2000)$ & $\delta(\mathbf{J} 2000)$ & Calibrator & & Calibrator & & \\
\hline 1994 Nov 17 & C & $X$ & 8.4649 & 0.100 & AR0323S & 053525.825 & -050951.38 & $3 \mathrm{C} 286$ & 5.06 & J0541-0541 & $1.240 \pm 0.010$ & 0.5 \\
\hline 1998 Jan 13 & $\mathrm{D}$ & $X$ & 8.4851 & 0.100 & AR0387 & 053524.220 & $\begin{array}{lll}-05 & 10 & 07.27\end{array}$ & $3 \mathrm{C} 48$ & 3.15 & J0541-0541 & $0.7420 \pm 0.0010$ & 1.0 \\
\hline 2000 Jan 14 & $\mathrm{~B}$ & $X$ & 8.4851 & 0.100 & AR0411 & 053526.970 & -051001.20 & $3 \mathrm{C} 48$ & 3.28 & J0541-0541 & $1.299 \pm 0.003$ & 3.2 \\
\hline 2014 Oct 16 & $\mathrm{C}$ & $K$ & 22.000 & 8.048 & AM1313 & 053527.070 & -051000.60 & $3 \mathrm{C} 147$ & 2.78 & J0541-0541 & $0.7188 \pm 0.0010$ & 1.4 \\
\hline 2014 Oct 16 & $\mathrm{C}$ & $Q$ & 44.063 & 8.048 & AM1313 & 053527.070 & -051000.60 & $3 \mathrm{C} 147$ & 0.91 & J0541-0541 & $0.5234 \pm 0.0006$ & 0.6 \\
\hline 2014 Oct 18 & $\mathrm{C}$ & $\vec{C}$ & 6.000 & 4.048 & AM1313 & 053527.070 & -051000.60 & $3 \mathrm{C} 147$ & 7.94 & J0541-0541 & $0.9260 \pm 0.0013$ & 0.5 \\
\hline 2014 Oct 18 & $\mathrm{C}$ & $X$ & 10.000 & 4.048 & AM1313 & 053527.070 & -051000.60 & $3 \mathrm{C} 147$ & 4.84 & J0541-0541 & $0.9050 \pm 0.0006$ & 0.5 \\
\hline 2015 Aug 06 & A & $C$ & 6.224 & 3.998 & AO0316 & 053527.080 & -051000.30 & $3 \mathrm{C} 147$ & 7.94 & J0541-0541 & $1.161 \pm 0.004$ & 0.5 \\
\hline 2015 Sep 11 & A & $C$ & 6.224 & 3.998 & AO0316 & 053527.080 & $-0510 \quad 00.30$ & $3 \mathrm{C} 147$ & 7.94 & J0541-0541 & $1.066 \pm 0.004$ & 0.5 \\
\hline
\end{tabular}


Table 2

Positions of the Radio Sources

\begin{tabular}{|c|c|c|c|c|c|}
\hline \multirow[b]{2}{*}{ Source } & \multirow{2}{*}{$\begin{array}{l}\text { Alternative } \\
\text { Names }\end{array}$} & \multirow[b]{2}{*}{ Nature } & \multirow[b]{2}{*}{ References } & \multicolumn{2}{|c|}{ Position $^{a}$} \\
\hline & & & & $\alpha(\mathrm{J} 2000)$ & $\delta(\mathrm{J} 2000)$ \\
\hline VLA 11 & FIR 3, HOPS 370, MIPS 2302, MIR 21 & Class I YSO & 1 & $053527.6337 \pm 0.0007$ & $-050934.368 \pm 0.011$ \\
\hline MIPS 2301 & MIR 22 & YSO & 2 & $053527.639 \pm 0.008$ & $-050937.09 \pm 0.12$ \\
\hline MIPS 2297 & MIR 23 & Class II YSO & 2 & $053527.4711 \pm 0.0020$ & $-050944.06 \pm 0.03$ \\
\hline VLA $12 \mathrm{~N}$ & & Jet knot & 3 & $053527.385 \pm 0.011$ & $-050950.68 \pm 0.16$ \\
\hline HOPS 64 & MIPS 2293, MIR 24, FIR 4d/e & Class I YSO & 1 & $053526.9960 \pm 0.0023$ & $-050954.06 \pm 0.03$ \\
\hline VLA $12 \mathrm{C}$ & & Jet knot & 3 & $053527.111 \pm 0.012$ & $-050958.57 \pm 0.18$ \\
\hline HOPS 108 & FIR 4, MIPS 2289 & Class 0 YSO & 1 & $053527.086 \pm 0.004$ & $-050959.95 \pm 0.06$ \\
\hline VLA $12 \mathrm{~S}$ & & Jet knot & 3 & $053526.9389 \pm 0.0017$ & $-051002.876 \pm 0.025$ \\
\hline VLA 16 & & YSO & 3 & $053526.824 \pm 0.003$ & $-051005.64 \pm 0.04$ \\
\hline VLA 15 & FIR $4 \mathrm{j}$ & YSO & 3 & $053526.4091 \pm 0.0011$ & $-051005.951 \pm 0.016$ \\
\hline
\end{tabular}

Note.

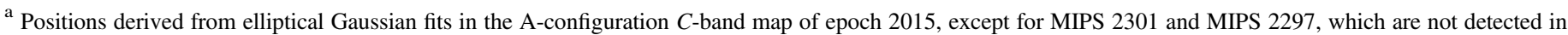

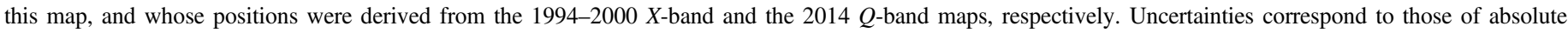

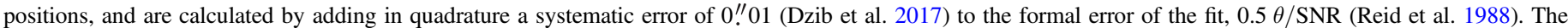

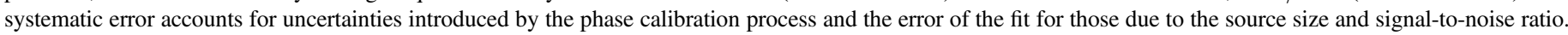
References. (1) Furlan et al. (2016), (2) Megeath et al. (2012), (3) This paper.

below). This rules out the $\mathrm{H}$ II region scenario proposed by López-Sepulcre et al. (2013) for VLA 12.

Although the position of the knot VLA $12 \mathrm{C}$ at 6 and $3 \mathrm{~cm}$ appears somewhat $(\sim 1$ !"5) displaced to the northeast of the position of HOPS 108 (as obtained from 24 and $8 \mu \mathrm{m}$ data; Megeath et al. 2012), at 1.3 and $0.7 \mathrm{~cm}$ the radio emission appears to be closer to the HOPS 108 IR position (see Figure 1). This shift in the position could indicate either an opacity gradient or that the radio emission observed at shorter wavelengths originates in a different object than the emission observed at longer wavelengths. This last suggestion is confirmed by our data of subarcsecond angular resolution at long wavelengths (Figure 4), which reveal two radio sources spatially separated by $\sim 2^{\prime \prime}$. We identify the northeasternmost of these two radio sources with the VLA 12C knot and the other source, whose position coincides with the IR source, with the radio counterpart of the HOPS 108 protostar. Recent ALMA observations with an angular resolution of 0 ." 12 (J. Tobin et al. $2017 \mathrm{~b}$, in preparation) reveal a compact source at $870 \mu \mathrm{m}$ whose position coincides within $<0$ !! 1 with the $\mathrm{cm}$ position (Table 3 ) confirming the nature of HOPS 108 as a protostar and its association with the $\mathrm{cm}$ radio source. Furthermore, the spectral index of HOPS 108 obtained from the centimeter data that separate its emission from that of VLA 12C shows that HOPS 108 has a positive spectral index $(\alpha=0.7 \pm 0.3$; Table 3 and Figure 3), thus it is associated with thermal (freefree) emission, in contrast to the knots of VLA 12, whose emission is non-thermal. From the empirical correlation between cm flux density and bolometric luminosity for protostellar objects, $\left(S_{\nu} d^{2} / \mathrm{mJy} \mathrm{kpc}^{2}\right)=0.008\left(L_{\text {bol }} / L_{\odot}\right)^{0.6}$ (Anglada et al. 2015), and using our measured flux density at $3 \mathrm{~cm}$ (Table 3), we estimate a bolometric luminosity $L_{\mathrm{bol}} \simeq 7 L_{\odot}$ for HOPS 108 . This value of the bolometric luminosity is close to the lowest end of the range considered by Furlan et al. (2016) in the modeling of this object, being much smaller than early estimates $\left(L_{\text {bol }} \simeq 1000 L_{\odot} ;\right.$ Crimier et al. 2009; López-Sepulcre et al. 2013) that were based on very low angular resolution data, and significantly smaller than the value obtained if the contribution of the VLA 12 knots is not removed $\left(L_{\text {bol }} \simeq 100 L_{\odot}\right.$; Furlan et al. 2014). Thus, separating the emission of HOPS 108 from that of the VLA 12 knots confirms HOPS 108 as a low-luminosity object.

The radio source VLA 11, which was associated with FIR 3 (HOPS 370) and reported as unresolved by Reipurth et al. (1999), is clearly resolved as a very elongated bipolar radio jet along a $\mathrm{PA} \simeq 5^{\circ}$ in our A-configuration data at $5 \mathrm{~cm}$ (Figure 4). The radio source is also resolved in the $3.6 \mathrm{~cm}$ image obtained by combining archive data from 1994 to 2000. The spectral index is $\alpha=0.27 \pm 0.10$ (Table 3, Figure 3), which is a reasonable value for a thermal free-free radio jet (Anglada 1996). HOPS 370 is known to be associated with an outflow extending along a PA similar to that of the radio jet (González-García et al. 2016; see Figure 2, left). The morphology of VLA 11 appears to change in the $0.7 \mathrm{~cm}$ image (Figure 1), which shows weak extended emission in a direction perpendicular to the jet, suggesting a noticeable dust contribution at this wavelength. To prevent dust contamination, the $0.7 \mathrm{~cm}$ data point has been excluded in the fits to determine the spectral index of this and the remaining thermal sources.

In the lower-resolution images at 6 and $3 \mathrm{~cm}$ (Figure 1), VLA 11 appears to be blended with the radio emission of MIPS 2301 (Megeath et al. 2012, an IR source also known as MIR 22 , Nielbock et al. 2003) located $\sim 3^{\prime \prime}$ south of VLA 11. MIPS 2301 is well separated, but only marginally detected, in the higher-resolution $3.6 \mathrm{~cm}$ image shown in Figure 4 and in the A-configuration images at $5 \mathrm{~cm}$ obtained with the robust weighting parameter set to values $>1$ (not shown in Figure 4). ${ }^{18}$ With the data currently available we obtain a positive spectral index, $\alpha=0.30 \pm 0.23$ (Table 3, Figure 3), consistent with thermal free-free emission from a YSO. More sensitive observations are required for a better characterization of the nature of the radio emission of this object.

The source MIPS 2297 (Megeath et al. 2012, also known as MIR 23; Nielbock et al. 2003) is marginally detected at $1.3 \mathrm{~cm}$ and $0.7 \mathrm{~cm}$ (Figure 1), but clearly detected in the $0.9 \mathrm{~cm}$ observations of J. Tobin et al. (2017a, in preparation). It shows a positive spectral index $(\alpha=1.5 \pm 0.5)$ in the centimeter

\footnotetext{
${ }^{18}$ Note that MIPS 2301 is not aligned along the VLA 11 jet axis $\left(\mathrm{PA}=5^{\circ}\right.$ ). Thus, its radio emission can be distinguished from the knots of the radio jet in the high angular resolution images.
} 

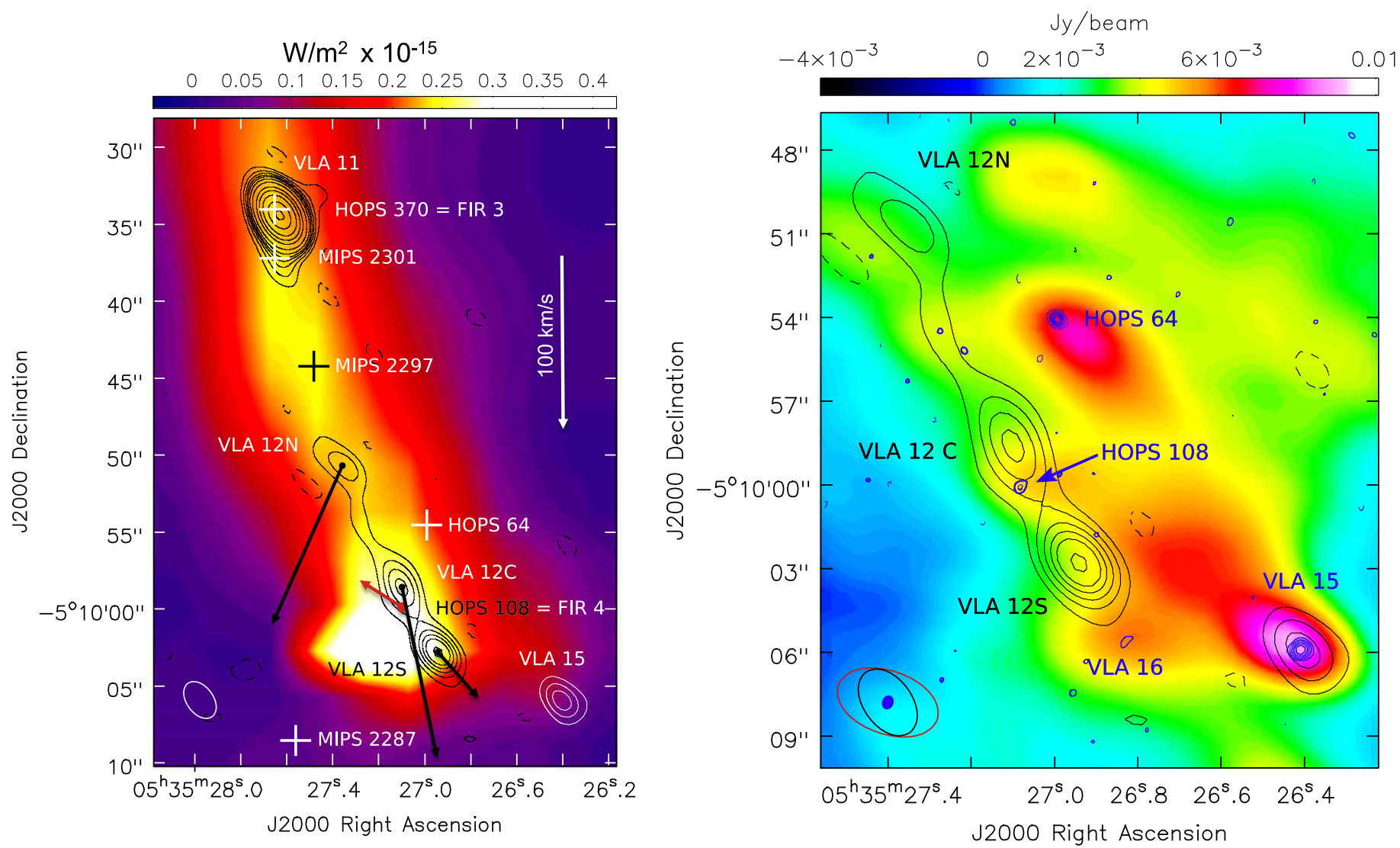

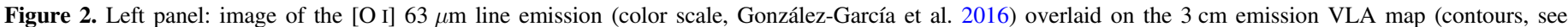

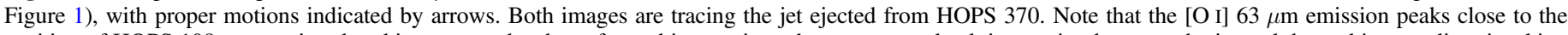

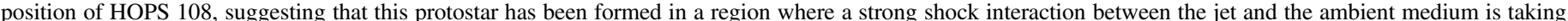

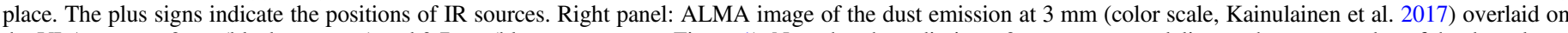

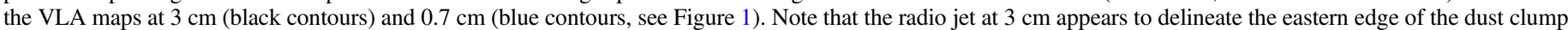
traced by the ALMA image.

regime (Table 3 and Figure 3), confirming its nature as a YSO. So far, MIPS 2297 has not been studied in great detail, but it is classified as a young star with a disk (Class II) by Megeath et al. (2012).

We also detect HOPS 64 (Figure 1), a Class I object (Furlan et al. 2016), which in the centimeter range presents a positive spectral index of $\alpha=1.51 \pm 0.06$ (Table 3, Figure 3), suggesting optically thick free-free emission from a YSO. The position of the radio source falls in between the FIR $4 \mathrm{~d}$ and FIR 4e cores imaged by Shimajiri et al. (2008), and is close to, but slightly displaced from, one of the main emission peaks of the dust cloud imaged by ALMA at $3 \mathrm{~mm}$ (Kainulainen et al. 2017; see Figure 2, right). Nevertheless, the position of the radio source coincides accurately (within $<0$ ". 1 ) with that of a compact $870 \mu \mathrm{m}$ source recently detected with ALMA (J. Tobin et al. 2017b, in preparation).

A few arcseconds to the southwest of the knot VLA 12S we detect a weak source. The source has not been reported previously at other wavelengths and we call it VLA 16. The detection is marginal in the individual bands ( $\sim 5 \sigma$ at most) but we consider that the source is real because it appears in four of the five observed bands (see Table 3 ). The spectral index is $\sim 0.7$ (Figure 3), consistent with that of a YSO/jet. The position of the radio source falls near a secondary emission peak in the $3 \mathrm{~mm}$ ALMA image (Kainulainen et al. 2017) shown in Figure 2 and coincides very well (within $<0$ ". 1 ) with the position of a weak $870 \mu \mathrm{m}$ source recently detected with
ALMA observations of higher angular resolution (J. Tobin et al. 2017b, in preparation). From the empirical correlation between centimeter emission and bolometric luminosity (Anglada et al. 2015), we infer $L_{\mathrm{bol}} \simeq 1 L_{\odot}$. All these results confirm the reality of the source VLA 16 and its nature as an embedded low-luminosity YSO.

Finally, we report the detection in all the observed bands of a compact source, located $\sim 8$ " $5 \mathrm{SW}$ of VLA $12 \mathrm{~S}$, and that we call VLA 15 (Tables 2, 3, Figures 1, 3, 4). The position of the source coincides with the FIR 4j core imaged by Shimajiri et al. (2008) and falls close to (but slightly offset from) one of the main emission peaks of the dust cloud imaged at $3 \mathrm{~mm}$ by ALMA (Kainulainen et al. 2017; see Figure 2, right). No counterparts at other wavelengths have been reported so far, but the source has been recently imaged at $870 \mu \mathrm{m}$ with ALMA (J. Tobin et al. $2017 \mathrm{~b}$, in preparation). The radio source has a positive spectral index $(\alpha=1.02 \pm 0.15)$ and we suggest that it traces a very young embedded stellar object associated with the OMC-2 starforming region that deserves further study at other wavelengths.

\subsection{Proper Motions of the Detected Sources}

We searched for proper motions by comparing the positions of the radio sources detected in the high angular resolution maps obtained from archival data (epochs 1994-2000) and from our last observations (epoch 2015). Unfortunately, the sensitivity of the archival data in a single epoch was insufficient, and we combined 


\begin{tabular}{|c|c|c|c|c|c|c|c|c|c|c|}
\hline \multirow[b]{2}{*}{ Source } & \multicolumn{5}{|c|}{ Flux Density $^{a}$} & \multirow[b]{2}{*}{$\begin{array}{l}\text { Spectral } \\
\text { Index }^{c}\end{array}$} & \multicolumn{4}{|c|}{ Proper Motions ${ }^{\mathrm{d}}$} \\
\hline & $\begin{array}{l}5 \mathrm{~cm} \\
(\mathrm{mJy})\end{array}$ & $\begin{array}{l}3 \mathrm{~cm} \\
(\mathrm{mJy})\end{array}$ & $\begin{array}{l}1.3 \mathrm{~cm} \\
(\mathrm{mJy})\end{array}$ & $\begin{array}{c}0.9 \mathrm{~cm}^{\mathrm{b}} \\
(\mathrm{mJy})\end{array}$ & $\begin{array}{c}0.7 \mathrm{~cm} \\
(\mathrm{mJy})\end{array}$ & & $\begin{array}{c}\mu_{\alpha} \cos \delta \\
\left(\mathrm{mas} \mathrm{yr}^{-1}\right)\end{array}$ & $\begin{array}{c}\mu_{\delta} \\
\left(\operatorname{mas~yr}^{-1}\right)\end{array}$ & $\begin{array}{c}V \\
\left(\mathrm{~km} \mathrm{~s}^{-1}\right)\end{array}$ & $\begin{array}{c}\text { P.A. } \\
\text { (degree) }\end{array}$ \\
\hline VLA 11 & $1.76 \pm 0.18$ & $2.16 \pm 0.22$ & $2.6 \pm 0.3$ & $2.6 \pm 0.3$ & $4.0 \pm 0.4$ & $0.27 \pm 0.10$ & $0.7 \pm 0.8$ & $0.7 \pm 0.8$ & $2 \pm 2$ & $50 \pm 50$ \\
\hline MIPS 2301 & $0.016 \pm 0.004^{\mathrm{e}}$ & $0.032 \pm 0.010^{\mathrm{f}}$ & $<0.03$ & $<0.03$ & $<0.07$ & $0.30 \pm 0.23$ & $\ldots g$ & $\ldots g$ & $\ldots \mathrm{g}$ & $\ldots g$ \\
\hline MIPS 2297 & $<0.03$ & $<0.03$ & $0.049 \pm 0.012$ & $0.092 \pm 0.013$ & $0.116 \pm 0.022$ & $1.4 \pm 0.5$ & $\ldots \mathrm{g}$ & $\ldots \mathrm{g}$ & $\ldots \mathrm{g}$ & $\ldots \mathrm{g}$ \\
\hline VLA $12 \mathrm{~N}$ & $0.134 \pm 0.020$ & $0.073 \pm 0.014$ & $<0.03$ & $<0.025$ & $<0.05$ & $-1.07 \pm 0.07$ & $22 \pm 11$ & $-46 \pm 11$ & $100 \pm 21$ & $155 \pm 12$ \\
\hline HOPS 64 & $0.011 \pm 0.003^{\mathrm{e}}$ & $<0.03$ & $0.069 \pm 0.012$ & $0.140 \pm 0.016$ & $0.30 \pm 0.03$ & $1.51 \pm 0.06$ & $\ldots \mathrm{g}$ & $\ldots \mathrm{g}$ & $\ldots \mathrm{g}$ & $\ldots \mathrm{g}$ \\
\hline VLA $12 \mathrm{C}$ & $0.24 \pm 0.03^{\mathrm{h}}$ & $0.09 \pm 0.03^{\mathrm{h}}$ & $<0.03$ & $<0.024$ & $<0.04$ & $-1.3 \pm 0.4$ & $-11 \pm 14$ & $-50 \pm 15$ & $100 \pm 30$ & $192 \pm 16$ \\
\hline HOPS 108 & $0.018 \pm 0.003^{\mathrm{e}}$ & $0.060 \pm 0.014^{\mathrm{f}}$ & $0.047 \pm 0.011$ & $0.115 \pm 0.014$ & $0.084 \pm 0.017$ & $0.7 \pm 0.3$ & $14 \pm 7$ & $9 \pm 7$ & $32 \pm 14$ & $57 \pm 25$ \\
\hline VLA $12 S$ & $0.25 \pm 0.03$ & $0.192 \pm 0.024$ & $0.16 \pm 0.03$ & $0.137 \pm 0.020$ & $<0.04$ & $-0.59 \pm 0.20$ & $-13 \pm 3$ & $-14 \pm 3$ & $37 \pm 6$ & $223 \pm 10$ \\
\hline VLA 16 & $0.017 \pm 0.003^{\mathrm{e}}$ & $<0.03$ & $0.040 \pm 0.010$ & $0.059 \pm 0.012$ & $0.069 \pm 0.014$ & $0.73 \pm 0.06$ & $\ldots \mathrm{g}$ & $\ldots \mathrm{g}$ & $\ldots{ }^{\mathrm{g}}$ & $\ldots \mathrm{g}$ \\
\hline VLA 15 & $0.071 \pm 0.014$ & $0.100 \pm 0.013$ & $0.200 \pm 0.022$ & $0.32 \pm 0.04$ & $0.60 \pm 0.07$ & $1.02 \pm 0.15$ & $-1 \pm 3$ & $2 \pm 3$ & $4 \pm 6$ & $340 \pm 90$ \\
\hline
\end{tabular}

Notes.

${ }^{a}$ Measured in the 2014 C-configuration maps, except when noted. Primary beam correction has been applied. Upper limits are $3 \sigma$.

${ }^{\mathrm{b}}$ From C-configuration observations made on 2016 March 27 (J. Tobin et al. 2017a, in preparation).

${ }^{\mathrm{c}}$ The data point at $0.7 \mathrm{~cm}$ has been excluded from the fit in the sources with positive spectral index to avoid possible contamination from dust emission.

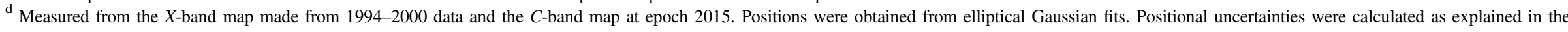

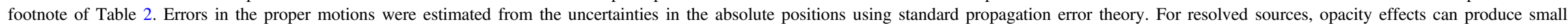
differences in the positions if they are obtained from maps at different wavelengths and/or angular resolutions, resulting in an additional uncertainty in the proper motion measurements.

${ }^{\text {e }}$ Obtained from the $C$-band 2015 A-configuration map because the source is not detected or appears blended with nearby sources in the $C$-band $2014 \mathrm{C}$-configuration map.

${ }^{\mathrm{f}}$ Obtained from the 1994-2000 multiconfiguration map at $3.6 \mathrm{~cm}$ because the source appears blended with nearby sources in the $X$-band $2014 \mathrm{C}$-configuration map.

$\mathrm{g}$ Not detected in the first epoch map (1994-2000 data) or too weak for a reliable positional fit.

${ }^{\mathrm{h}}$ The expected contribution of HOPS 108, estimated from higher-resolution data, has been subtracted. 

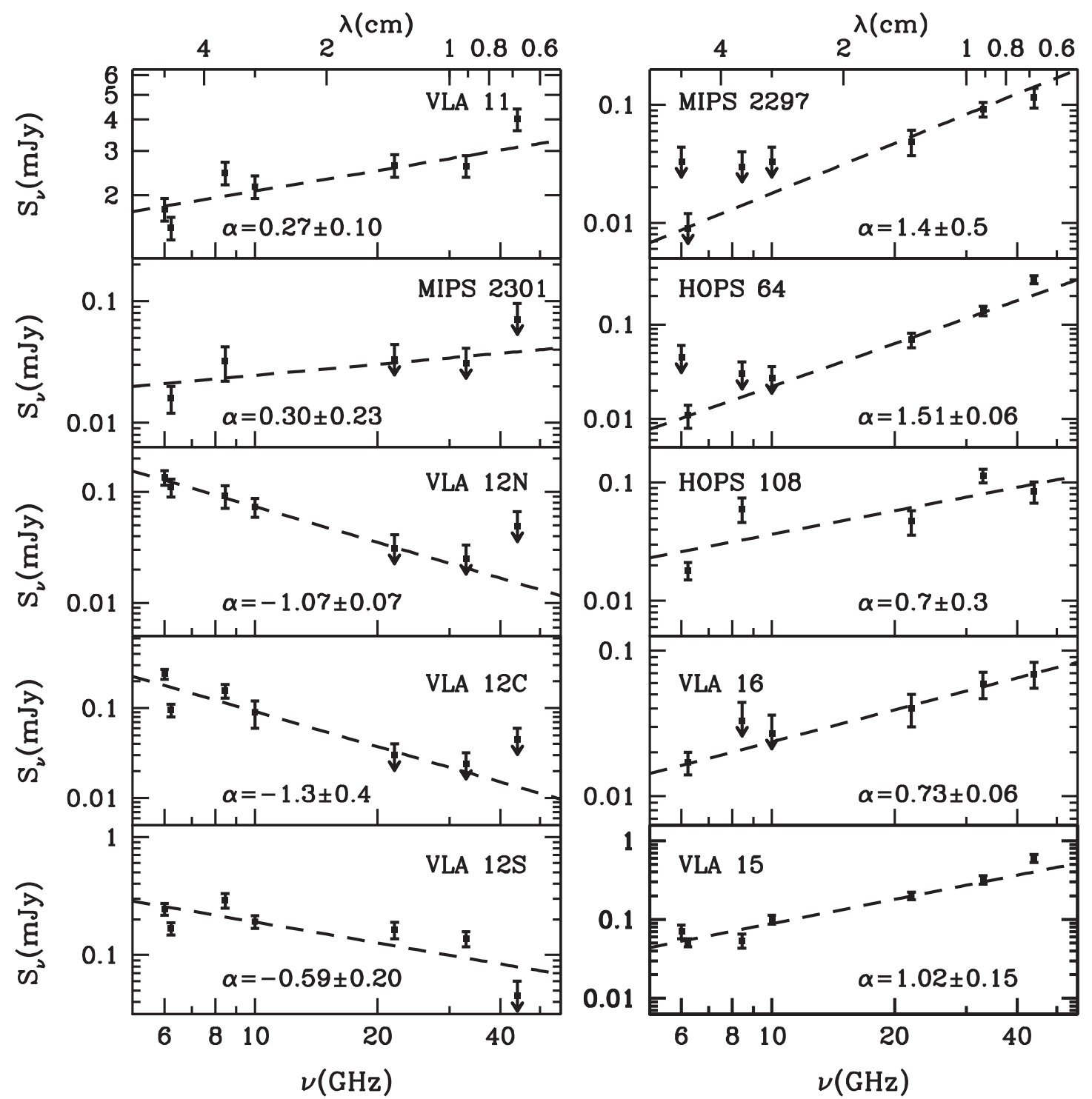

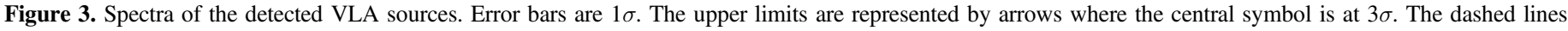

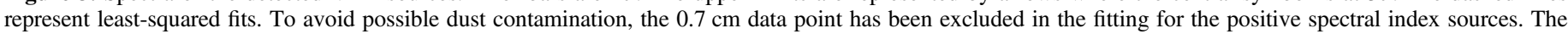
upper limits have been taken into account only when they constrain the fit.

data taken over $\sim 6$ years into the same map, which is not optimal to measure accurate proper motions, because of motions and changes in morphology of the sources over this period. Also, the archival data were observed in the $X$ band $(8.4-8.5 \mathrm{GHz})$, while the new data have been obtained in the $C$ band $(4-8 \mathrm{GHz})$ (Table 1). Although the frequency ranges of the two bands are almost contiguous, the differences in frequency could result in apparent motions due to variations of opacity with frequency, particularly in extended sources. Despite all these factors that contribute to the uncertainty in our measurements, the relatively high velocity of the jet and the amount of time ( $\sim 18$ years) that elapsed between the two maps make the proper motions clearly detectable. Our results show that, while VLA 11 and VLA 15 remain static within the uncertainties, the VLA $12 \mathrm{~N}$, VLA $12 \mathrm{C}$ and VLA 12S knots present measurable proper motions of $\sim 40-100 \mathrm{~km} \mathrm{~s}^{-1}$ roughly in the south direction (Table 3 and Figure 4; see also the left panel in Figure 2). HOPS 108 shows a measurable proper motion $\left(32 \pm 14 \mathrm{~km} \mathrm{~s}^{-1}\right)$, in the northeast direction, whose nature is uncertain (see below). The proper motion velocity of VLA 11 , the source with the best signal-to- noise ratio, is $2 \pm 2 \mathrm{~km} \mathrm{~s}^{-1}$ (Table 3). Since this velocity is obtained from the absolute positions of the source, its small value indicates that systematic errors are effectively very small and that their possible residual effects on the proper motion determination are at a level of $\sim 2 \mathrm{~km} \mathrm{~s}^{-1}$ or smaller.

\subsubsection{The HOPS 370 Radio Jet}

As discussed above, the knots of VLA 12 are characterized by negative spectral indices, indicating non-thermal emission (see Figure 3), but their overall morphology with three aligned knots suggests that they pertain to a radio jet. Radio jets associated with YSOs are characterized by thermal (free-free) emission (e.g., Anglada 1996), but in a few cases, non-thermal emission has been found in the jet lobes at relatively large distances from the jet core, which shows a positive spectral index and thermal emission (Carrasco-González et al. 2013; Anglada et al. 2015; RodríguezKamenetzky et al. 2016 and references therein). The most spectacular case of this selected sample of non-thermal radio jets is the $\mathrm{HH} 80$ radio jet, where linearly polarized emission, indicative 

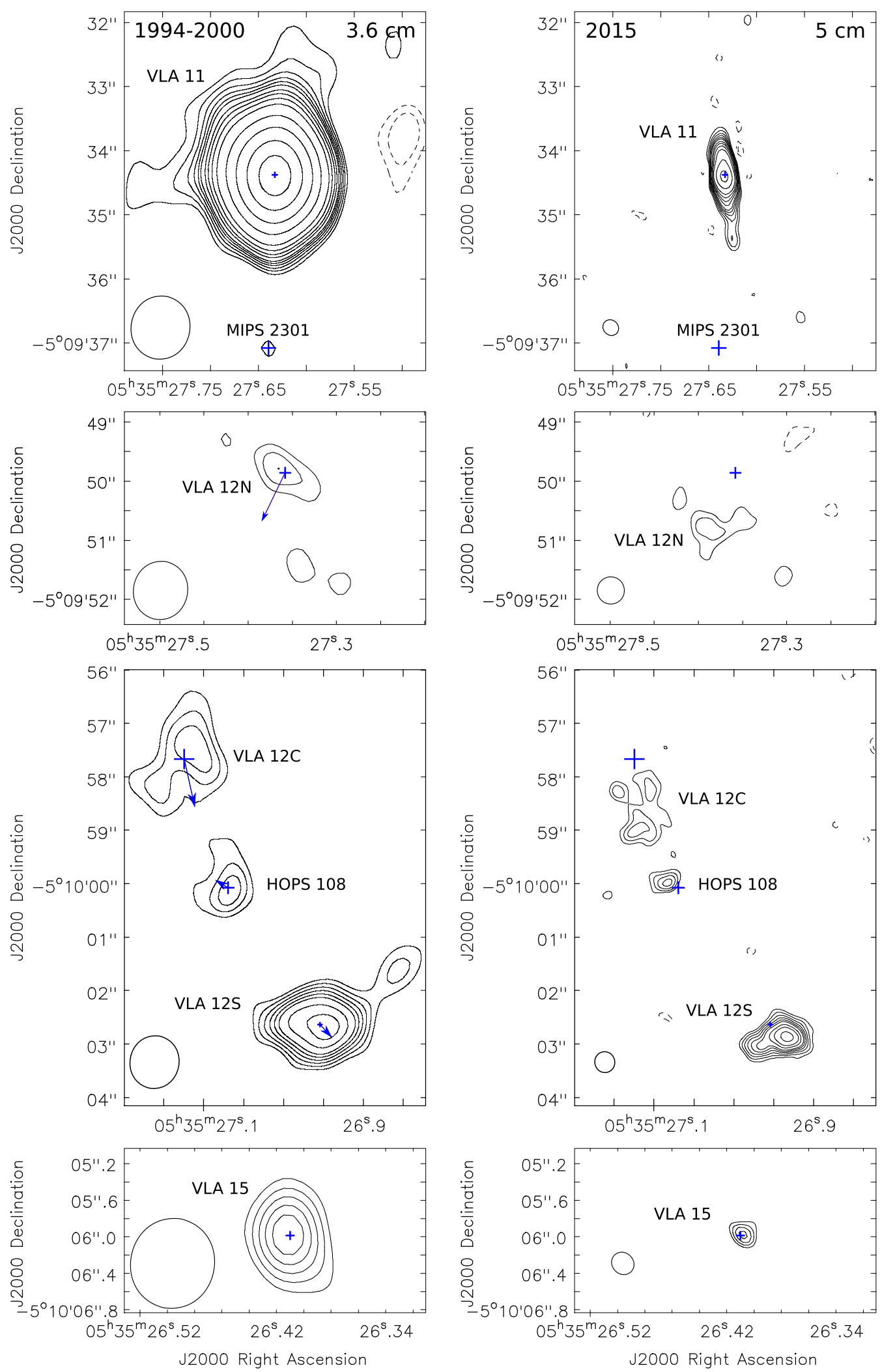

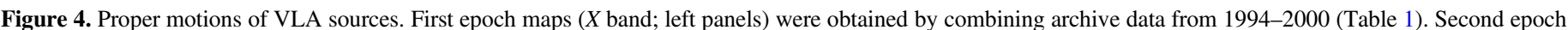

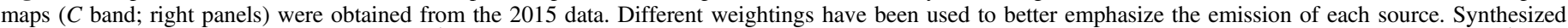

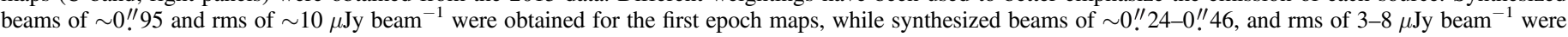

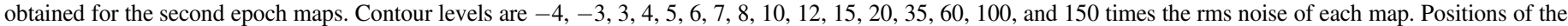

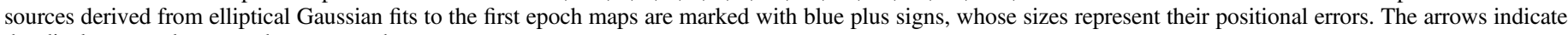
the displacement between the two epochs. 
of synchrotron radiation, was imaged in the jet lobes (CarrascoGonzález et al. 2010), while the central core of the jet, with a positive (thermal) spectral index, remained unpolarized. As the velocities of protostellar jets are not relativistic $\left(<1000 \mathrm{~km} \mathrm{~s}^{-1}\right)$, it is assumed that the synchrotron-emitting electrons are accelerated up to relativistic velocities via the diffusive shock acceleration mechanism (Drury 1991) working in the strong shocks present in the interaction of the jet with the surrounding medium. Thus, it is expected that the VLA 12 knots are associated with strong shocks, as indeed is suggested by the detection of several shock tracers in the proximity of this source (Shimajiri et al. 2008, Manoj et al. 2013, González-García et al. 2016; see Figure 2, left panel). Actually, the region near HOPS 108 shows the brightest far-IR [OI], $\mathrm{CO}, \mathrm{H}_{2} \mathrm{O}$, and $\mathrm{OH}$ line emission among the HOPS spectroscopic sample of sources (P. Manoj et al. 2017, in preparation), indicating the presence of strong shocks.

These results are consistent with the VLA 12 knots being part of a non-thermal radio jet either originating from the Class 0 protostar HOPS 108 (FIR 4), which falls in between the VLA $12 \mathrm{C}$ and the VLA $12 \mathrm{~S}$ knots, or from the intermediate-mass Class I YSO HOPS 370 (FIR 3), as the VLA 12 knots are roughly aligned in the direction of the VLA 11 radio jet associated with HOPS 370 (Figure 1) and fall on the southwest lobe of the outflow observed at submillimeter and far-IR wavelengths (Manoj et al. 2013; González-García et al. 2016) driven by this object.

If HOPS 108 was the driving source of the VLA 12 knots, one would expect proper motions of these knots away from HOPS 108. In particular, the VLA $12 \mathrm{~N}$ and $12 \mathrm{C}$ knots would show proper motions, with a component pointing to the north. However, as Figure 4 shows, the proper motions of the VLA 12 knots point to the south, consistent with an origin from HOPS 370 (VLA 11), and excluding HOPS 108 as the driving source. Thus, we conclude that the VLA 12 non-thermal radio knots belong to a radio jet, driven by the intermediate-mass Class I object HOPS 370, whose central thermal region is traced by the collimated radio source VLA 11 . VLA 11 is bipolar, extending $\sim 1^{\prime \prime}$ both to the northeast and to the southwest of the position of the protostar (see the top right panel in Figure 4). However, only the southwest side of the radio jet shows a distant $\left(\sim 30^{\prime \prime}\right)$ non-thermal lobe (VLA 12), without a northeast counterpart. The absence of a northeast non-thermal radio lobe is probably due to the lack of a dense clump along the NE jet path where a strong shock interaction, similar to that observed in the SW lobe, could take place. The jet is also bipolar in the far-IR [O I] lines (González-García et al. 2016), but its southwest lobe is more extended, with the brightest part associated with the nonthermal VLA 12 radio lobe.

Adopting 1997.65 as an average epoch for the archive data map, the resulting plane-of-the-sky velocities of the VLA $12 \mathrm{~N}$, $12 \mathrm{C}$, and $12 \mathrm{~S}$ knots are $\sim 100,101$, and $37 \mathrm{~km} \mathrm{~s}^{-1}$, respectively. The velocities of knots $12 \mathrm{~N}$ and $12 \mathrm{C}$ fall in the range of values measured for other protostellar radio jets (Anglada et al. 2015 and references therein); the velocity of VLA $12 \mathrm{~S}$ is smaller, suggesting that this distant knot could have suffered a significant deceleration, particularly if the jet has interacted strongly with the ambient medium, as indicated by other observations (Manoj et al. 2013, González-García et al. 2016). In this respect, recent proper motion measurements of the radio knots associated with the Herbig-Haro objects $\mathrm{HH} 80, \mathrm{HH} 81$, and $\mathrm{HH} 80 \mathrm{~N}$, far away from the central source, have shown that these knots have significantly slower motions than the radio knots located a few arcseconds from the central source (Masqué et al. 2015). This result has been interpreted as indicating strong jet interactions with the ambient cloud that significantly slow down the jet material.

\subsubsection{The Origin of HOPS 108}

Since the position of the HOPS 108 protostar falls in the path of the VLA 11-VLA 12 radio jet, but it can be ruled out as its driving source, an appealing alternative possibility is that the jet is related to the origin of HOPS 108. It has been proposed that shocks associated with the interaction of jets with the surrounding medium could compress the gas and induce local instabilities that may trigger star formation (e.g., Yokogawa et al. 2003; Graves et al. 2010; Duarte-Cabral et al. 2011). In particular, this scenario has been proposed for HOPS 108, as it is associated with strong shocks (Manoj et al. 2013; GonzálezGarcía et al. 2016) and is probably interacting with a nearby dense core (Shimajiri et al. 2008). Interestingly, the shape of the jet in the region of the VLA 12 knots appears to follow the eastern edge of a clump of enhanced dust emission, as imaged by ALMA at $3 \mathrm{~mm}$ (Kainulainen et al. 2017; see Figure 2, right panel). Thus, it seems plausible that the formation of the HOPS 108 protostar was triggered by the compression of the material in this clump after a strong shock interaction with the VLA 11VLA 12 jet, driven by HOPS 370 .

The evolutionary statuses of HOPS 370 and HOPS 108 are also fully consistent with the suggestion of the latter being triggered by the former. HOPS 370, with a bolometric temperature $T_{\text {bol }}=72 \mathrm{~K}$, has been classified as an intermediatemass young Class I object (Adams et al. 2012, Furlan et al. 2016) and we would expect an age (time elapsed since the onset of collapse) of a few times $10^{5}$ years for this object. HOPS 108, with a lower bolometric temperature $T_{\text {bol }}=38 \mathrm{~K}$, is supposed to be younger, and it has been classified as a Class 0 protostar (Adams et al. 2012, Furlan et al. 2016). We can then hypothesize that some $10^{5}$ years ago the jet of HOPS 370 started to impact the molecular clump (see Figure 2, right panel) from which HOPS 108 later formed. After an interaction of a few times $10^{4}$ years, enough time had passed to allow the collapse of the clump and the start of the protostellar stage of HOPS 108. The travel time of the gas from HOPS 370 to HOPS 108 is only 500 years (as derived from our measured proper motions) and the delay in the influence of the first object on the second can be taken to be much shorter than the other timescales. We then have a scenario in which the evolutionary status of the sources is consistent with one of them triggering the formation of the other.

It should be noted that the MIPS 2297 source also falls on the path of the HOPS 370 jet, but is closer to the origin. Since MIPS 2297 seems to be older than HOPS 108 one could think that it is the result of an earlier episode of triggered star formation. If MIPS 2297 is really a Class II object, as proposed by Megeath et al. (2012), it would be even older than HOPS 370, making the possibility of triggered formation inviable. However, MIPS 2297 is not yet well studied, and if it happens to be younger, this possibility cannot be fully discarded. On the other hand, Shimajiri et al. (2008) proposed that the formation of HOPS 64 ( $\sim 6^{\prime \prime}$ NW from HOPS 108 ) could have also been triggered by the outflow from HOPS 370 . However, in the recent study by Furlan et al. (2016), both HOPS 370 and HOPS 64 were classified as Class I sources, suggesting a similar evolutionary stage, which would be in conflict with a triggered star formation scenario for HOPS 64. It is also possible that MIPS 2297 and/or HOPS 64, which are both visible at shorter wavelengths, lie in 
front of the plane where HOPS 370 and HOPS 108 are located; so they could be close to HOPS 370 and its jet just in projection, but in reality be farther away and have formed in OMC-2 independently of this source. The new source VLA 16 also falls in the path of the jet, very close to the southernmost detected knot, VLA 12S. Also, we cannot exclude the possibility that star formation has been triggered, or will eventually be triggered, in other dust cores reported by Shimajiri et al. (2008), which currently do not show evidence of embedded YSOs. Thus, there are at least four young stars that could be the result of triggering or be affected by the HOPS 370 jet, but only for HOPS 108 is there further evidence of ongoing shock interaction.

A puzzling issue in the triggered formation scenario for HOPS 108 is its large proper motion velocity $\left(\sim 32 \mathrm{~km} \mathrm{~s}^{-1}\right.$; Table 3$)$. Such a large velocity implies that it should have been acquired very recently ( $\lesssim 200$ year) in order for the star to be formed in the proximity (within a few arcsec) of its current position, in the region where the shock emission has been identified (Manoj et al. 2013; González-García et al. 2016). Otherwise, if this velocity was maintained during a longer time interval, it would imply that the HOPS 108 protostar originated at a distant position from its current location, making the triggering hypothesis inviable. Relatively low velocities are expected if the protostars are mechanically linked to the molecular gas reservoir and thus obtain their velocities directly from the gas they are forming in, as proposed by Stutz \& Gould (2016), who found typical radial velocities with respect to the ambient cloud of $\sim 0.6 \mathrm{~km} \mathrm{~s}^{-1}$ for protostars and $\sim 1.8 \mathrm{~km} \mathrm{~s}^{-1}$ for pre-mainsequence stars in the integral-shaped filament region in Orion. These authors propose a slingshot-like ejection mechanism to account for the increase in velocity of pre-main-sequence stars relative to protostars. However, this mechanism cannot explain velocities above a few $\mathrm{km} \mathrm{s}^{-1}$.

In contrast, HOPS 108 presents a much larger proper motion velocity, similar to the $\sim 27 \mathrm{~km} \mathrm{~s}^{-1}$ of the $\mathrm{BN}$ object in the nearby Orion BN/KL stellar system (Goddi et al. 2011; Rodríguez et al. 2017). Another runaway star, V 1326 Ori, with a peculiar large proper motion velocity of about $28 \mathrm{~km} \mathrm{~s}^{-1}$ has been recently identified in a radio survey of proper motions in the core of the Orion Nebula Cluster (Dzib et al. 2017). For the $\mathrm{BN} / \mathrm{KL}$ system, in order to explain both the uncollimated explosive molecular outflow (Zapata et al. 2009) and the stellar velocities, violent (proto-)stellar interactions and the subsequent dynamical ejection of said (proto-)stars have been invoked (Bally \& Zinnecker 2005; Gómez et al. 2008; Bally et al. 2017). This acceleration mechanism would require the presence of a binary or multiple stellar system near FIR 4/ HOPS 108 to make it compatible with the triggering scenario. Shimajiri et al. (2008) imaged 11 potential cores within a region of $\sim 20^{\prime \prime} \times 20^{\prime \prime}$ in the proximity of FIR 4 and interpreted them as tracing a young stellar cluster whose formation was triggered by the impact of the jet with the ambient cloud. If this was the case, interactions within this multiple system could have resulted in the recent ejection of HOPS 108. We identify the region imaged by Shimajiri et al. (2008) with the clump of dust imaged by ALMA at $3 \mathrm{~mm}$ (Kainulainen et al. 2017; Figure 2, right panel), where the sources HOPS 108, HOPS 64, VLA 15 and VLA 16 appear to be embedded. Since HOPS 108 moves toward the northeast, we do not find a suitable candidate that might be receding from a close enough common position and that could be responsible for a past interaction with HOPS 108 , casting doubt on this possibility.
There are other detected radio sources in the region whose proper motions are compatible with receding from a common location with HOPS 108 (e.g., VLA 13, located $40^{\prime \prime}$ to the southwest; A. K. Díaz-Rodríguez et al. 2017, in preparation), and that could have dynamically interacted with it in the past. However, the location of a potential encounter would fall at a large distance from the current position of HOPS 108, and as noted above, would make the origin of this object incompatible with a scenario of (local) triggered star formation. On the other hand, if HOPS 108 was accelerated in a distant encounter it seems unlikely that our observation occurs just at the moment when it crosses the VLA 12 knots of the HOPS 370 jet, at the point of its maximum interaction with the ambient cloud.

Another possibility to explain the observed large proper motion of HOPS 108 is to assume that it is due to a change in the shape of its radio emission because of blending with the emission of the nearby VLA 12 knots. Since these knots move toward the southwest, with VLA 12C approaching HOPS 108 and VLA 12S going away, a partial blending with these knots can result in an apparent motion of HOPS 108 toward the northeast. Also, a one-sided ejecta of a high-velocity cloud of ionized plasma by the protostar could produce a change in the shape of its radio emission and an apparent proper motion. One-sided high velocity ejecta from young stars have been observed in several other sources (e.g., Rodríguez et al. 2017 and references therein). Such an ejecta with a velocity of the order of hundreds of $\mathrm{km} \mathrm{s}^{-1}$ in a poorly resolved source would result in a one-sided distortion in the source shape and thus a change in the centroid position that could mimic a motion of several tens of $\mathrm{km} \mathrm{s}^{-1}$. Wavelength-dependent opacity effects in an extended source could emphasize these changes in the position of the centroid of the emission. Indeed, we found that the precise position of the HOPS 108 radio source changes with wavelength. Additional, sensitive high angular resolution observations could provide a more precise measure of the HOPS 108 proper motions and reveal the possible presence of either a high-velocity ejecta from this source or a nearby binary/multiple system whose past interaction with HOPS 108 had been responsible for its current high proper motions.

\section{Conclusions}

We analyzed new (2014-2015) multiwavelength $(0.7-5 \mathrm{~cm})$, multiconfiguration VLA observations together with archival (1994-2000) data at $3.6 \mathrm{~cm}$ that allowed us to obtain spectral indices and proper motions of radio sources in the region enclosing FIR 3 (HOPS 370) and FIR 4 (HOPS 108) in OMC-2. Our main conclusions can be summarized as follows.

1. We detect radio emission from the far-IR sources HOPS 370, HOPS 108, and HOPS 64, as well as from the mid-IR sources MIPS 2297 and MIPS 2301. We also detect two new sources, both associated with dust cores, that we call VLA 15 and VLA 16. For all these sources we obtain positive spectral indices in the centimeter wavelength range, consistent with thermal free-free emission from YSOs.

2. The radio source VLA 11, associated with HOPS 370, presents a clearly elongated bipolar morphology in the northeast-southwest direction. Because of this morphology and positive spectral index $(\alpha \simeq 0.3)$ we interpret VLA 11 as the thermal region closest to the origin of a well-collimated bipolar radio jet driven by the HOPS 370 intermediate-mass protostar. We detect several knots of 
emission (VLA 12N, 12C, and 12S) at $\sim 7500-12,500$ au to the southwest of HOPS 370 with negative spectral indices and showing proper motions of $\sim 40-100 \mathrm{~km} \mathrm{~s}^{-1}$ away from the HOPS 370 protostar. We interpret these knots as a non-thermal lobe of the HOPS 370 jet. The VLA 12 knots are found in a region where previous observations detected several tracers of strong shocks, suggesting that their non-thermal emission is likely synchrotron emission from relativistic electrons accelerated in shocks, as has been proposed for other nonthermal jet lobes. Although VLA 11 is bipolar, a similar non-thermal radio lobe is not found to the northeast of HOPS 370 , probably because there is not a dense clump where the jet interaction could take place.

3. HOPS 108 is identified as a compact radio source that is independent and kinematically separated from the radio emission of the VLA 12 knots of the HOPS 370 jet. Its position along the path of the VLA 12 knots, and its coincidence with different shock tracers, suggest a scenario in which the formation of HOPS 108 has been triggered by the interaction of the HOPS 370 jet with the surrounding medium, as was already proposed by Shimajiri et al. (2008). The more advanced evolutionary stage of HOPS 370 relative to HOPS 108, and the short dynamical timescale of the jet, are consistent with this scenario. However, HOPS 108 presents a large proper motion velocity of about $30 \mathrm{~km} \mathrm{~s}^{-1}$ toward the NE whose nature is uncertain. This velocity is similar to the proper motion velocities found in runaway stellar sources in Orion (Dzib et al. 2017; Rodríguez et al. 2017). Such a large velocity in HOPS 108 would be inconsistent with the triggered scenario, unless the source had been accelerated to this high velocity very recently. Alternatively, an apparent proper motion could result because of a change in the position of the centroid of the source due to a partial blending with the emission of the nearby VLA 12 knots or to a one-sided ejecta of ionized plasma, rather than by the actual motion of the protostar itself. Deep high angular resolution observations at several epochs are required to clarify this issue.

4. HOPS 370 and VLA 15 do not show detectable proper motions $\left(V \lesssim 2-4 \mathrm{~km} \mathrm{~s}^{-1}\right)$, as expected for embedded protostars.

A.K.D.R. acknowledges a Spanish MECD FPU fellowship. G.A., A.K.D.R., J.F.G., and M.O. acknowledge support from MINECO (Spain) AYA2014-57369-C3-3-P grant (co-funded by FEDER). A.S. is thankful for funding from the "Concurso Proyectos Internacionales de Investigacion, Convocatoria 2015" (project code PII20150171) and the BASAL Centro de Astrofisica y Tecnologias Afines (CATA) PFB-06/2007. The authors thank Alvaro Sanchez-Monge for helpful suggestions and Jouni Kainulainen for providing the $3 \mathrm{~mm}$ ALMA image of this region.
Facility: VLA.

Software: CASA (v 4.2.2), AIPS.

\section{References}

Adams, J. D., Herter, T. L., Osorio, M., et al. 2012, ApJL, 749, L24

Anglada, G. 1996, in ASP Conf. Ser. 93, Radio Emission from the Stars and the Sun, ed. A. R. Taylor \& J. M. Paredes (San Francisco, CA: ASP), 3

Anglada, G., Rodríguez, L. F., \& Carrasco-Gonzalez, C. 2015, Advancing Astrophysics with the Square Kilometre Array (Trieste: SISSA), 121

Bally, J., Ginsburg, A., Arce, H., et al. 2017, ApJ, 837, 60

Bally, J., \& Zinnecker, H. 2005, AJ, 129, 2281

Briggs, D. S. 1995, BAAS, 27, 1444

Carrasco-González, C., Rodríguez, L. F., Anglada, G., et al. 2010, Sci, 330, 1209

Carrasco-González, C., Rodríguez, L. F., Anglada, G., et al. 2013, EPJC, 61, 03003

Chini, R., Reipurth, B., Ward-Thompson, D., et al. 1997, ApJL, 474, L135

Conway, J. E., Cornwell, T. J., \& Wilkinson, P. N. 1990, MNRAS, 246, 490

Crimier, N., Ceccarelli, C., Lefloch, B., \& Faure, A. 2009, A\&A, 506, 1229

Drury, L. O. 1991, MNRAS, 251, 340

Duarte-Cabral, A., Dobbs, C. L., Peretto, N., \& Fuller, G. A. 2011, A\&A, 528, A50

Dzib, S. A., Loinard, L., Rodríguez, L. F., et al. 2017, ApJ, 834, 139

Furlan, E., Fischer, W. J., Ali, B., et al. 2016, ApJS, 224, 5

Furlan, E., Megeath, S. T., Osorio, M., et al. 2014, ApJ, 786, 26

Goddi, C., Humphreys, E. M. L., Greenhill, L. J., Chandler, C. J., \& Matthews, L. D. 2011, ApJ, 728, 15

Gómez, L., Rodríguez, L. F., Loinard, L., et al. 2008, ApJ, 685, 333

González-García, B., Manoj, P., Watson, D. M., et al. 2016, A\&A, 596, A26

Graves, S. F., Richer, J. S., Buckle, J. V., et al. 2010, MNRAS, 409, 1412

Kainulainen, J., Stutz, A. M., Stanke, T., et al. 2017, A\&A, 600, A141

Kim, M. K., Hirota, T., Honma, M., et al. 2008, PASJ, 60, 991

Kounkel, M., Hartmann, L., Loinard, L., et al. 2017, ApJ, 834, 142

Lis, D. C., Serabyn, E., Keene, J., et al. 1998, ApJ, 509, 299

López-Sepulcre, A., Taquet, V., Sánchez-Monge, Á, et al. 2013, A\&A, 556, A62

Manoj, P., Watson, D. M., Neufeld, D. A., et al. 2013, ApJ, 763, 83

Masqué, J. M., Rodríguez, L. F., Araudo, A., et al. 2015, ApJ, 814, 44

Megeath, S. T., Gutermuth, R., Muzerolle, J., et al. 2012, AJ, 144, 192

Menten, K. M., Reid, M. J., Forbrich, J., \& Brunthaler, A. 2007, A\&A, 474,515

Mezger, P. G., Wink, J. E., \& Zylka, R. 1990, A\&A, 228, 95

Nielbock, M., Chini, R., \& Müller, S. A. H. 2003, A\&A, 408, 245

Peterson, D. E., \& Megeath, S. T. 2008, in Handbook of Star-forming Regions, ed. B. Reipurth (San Francisco, CA: ASP), 590

Reid, M. J., Schneps, M. H., Moran, J. M., et al. 1988, ApJ, 330, 809

Reipurth, B., Rodríguez, L. F., \& Chini, R. 1999, AJ, 118, 983

Rodríguez, L. F., Dzib, S. A., Loinard, L., et al. 2017, ApJ, 834, 140

Rodríguez, L. F., Marti, J., Canto, J., Moran, J. M., \& Curiel, S. 1993, RMxAA, 25, 23

Rodríguez-Kamenetzky, A., Carrasco-González, C., Araudo, A., et al. 2016, ApJ, 818, 27

Shimajiri, Y., Takahashi, S., Takakuwa, S., Saito, M., \& Kawabe, R. 2008, ApJ, 683, 255

Stutz, A. M., \& Gould, A. 2016, A\&A, 590, A2

Takahashi, S., Saito, M., Ohashi, N., et al. 2008, ApJ, 688, 344

Tsujimoto, M., Koyama, K., Kobayashi, N., et al. 2003, AJ, 125, 1537

Williams, J. P., Plambeck, R. L., \& Heyer, M. H. 2003, ApJ, 591, 1025

Yokogawa, S., Kitamura, Y., Momose, M., \& Kawabe, R. 2003, ApJ, 595, 266

Zapata, L. A., Schmid-Burgk, J., Ho, P. T. P., Rodríguez, L. F., \& Menten, K. M. 2009, ApJL, 704, L45 